\title{
INDICADORES: CONCEITO E COMPLEXIDADE DO MENSURAR EM ESTUDOS DE FENÔMENOS SOCIAIS
}

VALDECIR SOLIGO

\section{RESUMO}

O presente artigo objetiva debater a categoria "indicadores", focando o desenvolvimento histórico conceitual dos indicadores sociais e aspectos possíveis de relacionamento direto ou indireto com indicadores de qualidade da educação. Para tanto, faz-se uma breve incursão pelo processo de construção do campo teórico dos indicadores sociais para, posteriormente, analisar os Índices de Desenvolvimento da Educação Básica nos municípios catarinenses com menos de cinco mil habitantes e que participaram da Prova Brasil nos ciclos de 2005, 2007 e 2009. 0 recorte espaço-temporal está relacionado à raridade de pesquisas que visam à construção de significados para os indicadores no contexto da realidade dos pequenos municípios brasileiros. Como resultado preliminar, destaca-se a fragilidade da relação entre indicadores sociais e educacionais, diante da complexidade conceitual e de mensuração de fenômenos sociais que exigem a reinserção, da parte mensurável, na realidade sócio-histórica da qual foi retirada para compreensão e significação política e social.

PALAVRAS-CHAVE INDICADORES • MENSURAÇÃO • EDUCAÇÃO. 


\section{RESUMEN}

Este trabajo tiene como objetivo discutir la categoría "indicadores", centrándose en el desarrollo histórico conceptual de los indicadores sociales y en aspectos posibles que tengan una relación directa o indirecta con indicadores de calidad de la educación. Para ello, se hace una breve incursión en el proceso de construcción del campo teórico de los indicadores sociales para analizar, posteriormente, los índices de desarrollo de la educación básica en los municipios de Santa Catarina con menos de cinco mil habitantes que participaron de la Prueba Brasil en 2005, 2007 y 2009. El recorte espacio temporal está relacionado con la escasez de estudios dirigidos a la construcción de significados para los indicadores en el contexto de la realidad de los pequeños municipios brasileños. Como resultado preliminar, se destaca la fragilidad de la relación entre los indicadores sociales y educativos, frente a la complejidad conceptual y de medición de los fenómenos sociales que exigen la reinserción de lo mensurable en la realidad socio-histórica de la que fue retirada para la comprensión y la significación política y social.

PALABRAS CLAVE INDICADORES - MEDICIÓN • EDUCACIÓN.

\section{ABSTRACT}

This paper aims to discuss the category of "indicators" which focuses on the historical development of social indicators and conceptual aspects of possible direct or indirect relationship with quality indicators in education. Therefore, it is a brief incursion into the process of constructing a theoretical field concerning social indicators to further analyze the Basic Education Development indices in the municipalities of Santa Catarina with less than five thousand people and who took the Brazil Test in 2005, 2007 and 2009. The space-time perspective is related to the scarcity of studies aimed at the construction of meanings for the indicators in the context of small Brazilian municipalities. As a preliminary result, we highlight the fragility of the relationship between social and educational indicators in face of the conceptual and the measurement complexity of social phenomena which require the reinsertion of the measurable part in the sociohistorical reality from which it was taken for political and social understanding and significance.

KEYWORDS INDICATORS - MEASUREMENT - EDUCATION. 


\section{O CONCEITO E A CONSTRUÇÃO DO INDICADOR}

A mensuração da qualidade de vida e do desenvolvimento social, econômico e político vem adquirindo importância, à medida que essas informações tornam-se mais acessíveis a governos e população em geral. Diariamente, uma enxurrada de indicadores invade nossa vida. Medir e transformar essas medidas em índices utilizados para revelar e sinalizar diversos aspectos da sociedade passou a integrar inúmeras atividades cotidianas. No entanto, os fenômenos estudados pelas ciências sociais são demasiadamente complexos para serem interpretados e analisados sob uma ótica unidimensional. Para interpretar um fenômeno social, é necessário considerá-lo na sua multiplicidade de aspectos, procurando suas várias dimensões analíticas.

No início da história e da evolução dos indicadores, predominava a exclusiva quantificação, seja de pessoas, recursos ou equipamentos. A partir de 1920, e particularmente depois da Segunda Guerra Mundial, a estatística mudou completamente, saindo das mãos exclusivas de cientistas econômicos e passando a ser produzida por departamentos, agências e divisões de repartições públicas nacionais. 
Já o desenvolvimento de indicadores sociais é mais recente. Ainda que nos anos 1920 e 1930 possam ser identificadas contribuições significativas para a construção de um marco conceitual, foi na década de 1960 que ocorreram tentativas mais sistemáticas de organização e acompanhamento das transformações sociais e aferição do impacto das políticas sociais nas sociedades desenvolvidas e subdesenvolvidas. Nesse contexto, começaram a se avolumar evidências do descompasso entre crescimento econômico e melhoria das condições sociais da população, principalmente em países subdesenvolvidos. Uma dessas evidências está no indicador Produto Interno Bruto (PIB), que, apesar do aumento significativo em alguns países, verificava-se a persistência de altos níveis de pobreza e acentuavam-se as desigualdades sociais. Esse indicador era usado, até então, para demonstrar o nível de desenvolvimento socioeconômico, no entanto mostrava-se cada vez menos apropriado (CARLEY, 1985).

Instituições multilaterais como OCDE, Unesco, FAO, OIT, OMS, Unicef e Divisão de Estatística das Nações Unidas, reconhecendo os limites dos indicadores puramente econômicos, passaram a empreender esforços conceituais e metodológicos para desenvolvimento de instrumentos de mensuração do bem-estar e da mudança social. Somados a isso, os livros Social indicators (1967), de Bauer, e Toward a social report (1966), publicação oficial do governo dos Estados Unidos, desenvolvidos sob encomenda do governo estadunidense, representaram marcos importantes no processo que ficou conhecido, na década de 1960, como "Movimento de Indicadores Sociais".

Em muitos países os setores de estatísticas públicas incorporaram novas dimensões investigativas e passaram a produzir relatórios sociais de forma sistemática, acreditando que, com sistemas abrangentes de indicadores sociais, seria possível organizar melhor as ações dos governos, o que proporcionaria níveis crescentes de bem-estar social (BAUER, 1967).

O crescente destaque dado ao planejamento governamental e, por conseguinte, à estatística gerou expectativas para além do possível em curto e médio prazos. Os insucessos, sucessos parciais e excessos do planejamento tecnocrático criaram, nos anos 1970, o revés em relação à década anterior, gerando uma espécie de ceticismo tanto com o planejamento público, quanto com a finalidade e utilidade dos sistemas de indicadores sociais (HAKKERT, 1996). 
A década de 1980 foi palco de experiências de formulação e implementação de políticas públicas, como os planejamentos local e participativo, diminuindo o descrédito dos indicadores sociais e restabelecendo a pertinência instrumental. Universidades, sindicatos, centros de pesquisa e agências vinculadas ao sistema de planejamento público passaram a desenvolver esforços para aprimoramento conceitual e metodológico de instrumentos mais específicos de quantificação e qualificação das condições de vida, da pobreza estrutural e outras dimensões da realidade social (JANNUZZI, 2004).

A partir da Constituição de 1988 e no contexto da descentralização administrativa e tributária em favor dos municípios, vem crescendo a demanda por informações sociais. Cada vez mais, estados e municípios fazem uso de uma série de indicadores no planejamento e execução de políticas públicas, constituindo um aporte de grande utilidade e considerável aceitabilidade.

A complexidade dos debates em torno da qualidade da educação é outro exemplo da multiplicidade de aspectos sociais, políticos e econômicos envolvidos na medição da qualidade e do desempenho de alunos, escolas e sistemas de ensino. Segundo Colman e Nixson (1981), a medida direta do desenvolvimento de um fenômeno social seria impraticável, sendo necessária a utilização de indicadores para o desempenho dessa tarefa.

Os indicadores empregados para medição dos fenômenos sociais são conhecidos por "indicadores sociais" ou "indicadores socioeconômicos", que, por sua vez, incluem os indicadores educacionais. Os indicadores quantitativos englobam todas as formas de medição dos fenômenos estudados pelas diversas ciências praticadas pelo homem.

Essas ferramentas surgiram com as ciências naturais, a partir da construção de modelos específicos, apresentando resultados satisfatórios devido ao seu caráter determinístico. Já no caso das ciências sociais, as relações se dão de forma estocástica, pois não há ligação direta entre causa e efeito, em que o surgimento da causa apenas aumenta a possibilidade de o efeito ocorrer (TRZESNIAK, 1998).

Em função dessa limitação, os indicadores sociais não possuem o mesmo grau de consenso e nem a mesma força explicativa daqueles utilizados nas ciências exatas. Sua efetividade para explicação dos fenômenos analisados difere no espaço 
e no tempo. Um indicador útil em determinado lugar e época pode não ser proveitoso para outra região, ou até mesmo para a própria região em outro momento. Segundo Jannuzzi (2004, p. 15), indicador social:

É uma medida em geral quantitativa dotada de significado social substantivo, usado para substituir, quantificar ou operacionalizar um conceito social abstrato, de interesse teórico (para pesquisa acadêmica) ou programático (para formulação de políticas). É um recurso metodológico, empiricamente referido, que informa algo sobre um aspecto da realidade social ou sobre mudanças que estão se processando na mesma.

À medida que os conceitos se modificam, tanto no espaço quanto no tempo, os indicadores utilizados para qualificar esses conceitos também se modificam. Assim, ao se construir um indicador, dois pontos emergem como fundamentais. O primeiro é a escolha de qual aspecto da realidade será tratado. O segundo é definir qual o conceito abstrato que será utilizado para explicação do fenômeno estudado.

Da mesma forma, ao se analisarem indicadores, faz-se necessário atentar para o desenvolvimento de tais conceitos e valores. Para Colman e Nixson (1981), o desenvolvimento de um indicador pode ser considerado um processo de aperfeiçoamento em relação a um conjunto de valores ou enquanto comparação a esses valores.

Outro aspecto a se considerar, segundo Sen (2005), é que cada abordagem avaliatória pode ser caracterizada de acordo com sua base informacional. Tanto as informações que são coletadas para formar juízo, quanto aquelas desprezadas ou excluídas de um papel avaliatório direto, dizem respeito ao processo de construção do indicador e à abordagem escolhida.

Com o crescimento da visibilidade de inúmeras questões sociais, como distribuição de renda, miséria, mortalidade infantil, criminalidade, qualidade da educação, entre outras, os dados estatísticos passam a fazer parte do monitoramento das ações governamentais pela sociedade civil. Isso gera a necessidade da produção de informações que permitam um acompanhamento das mudanças da realidade social da população. 
A utilização da informação como instrumento de interpretação da realidade social requer a criação de meios de quantificação de alguns aspectos importantes dessa realidade de forma clara e simplificada. Isso não significa que um indicador possa ser produzido sem critérios científicos rígidos. Para tanto, Scheerens (2004), ao discutir a questão da melhoria da eficácia das escolas, destaca três características dos indicadores: mensuralidade, o que pode ser medido; centralidade, referente a aspectos essenciais que descrevem a situação no momento da coleta de dados; e padronização, que reflete aspectos da qualidade do objeto avaliado em relação a um padrão referente por intermédio de normas que permitem realizar juízos de valor. Assim, para a educação, os indicadores são estatísticas que possibilitam formular avaliações sobre aspectos essenciais do funcionamento dos sistemas educativos.

Já para Takashina (1999), os indicadores são representações quantificáveis das características de produtos e processos, sendo assim utilizados para a melhoria da qualidade e desempenho de um produto, serviço ou processo, ao longo do tempo. Os indicadores surgem como auxiliadores nas tomadas de decisões, fundamentando as argumentações mediante o fornecimento das informações dos processos. Assim, na criação de um indicador, a seletividade, a simplicidade, a clareza, a abrangência, a rastreabilidade, a acessibilidade, a comparabilidade, a estabilidade, a rapidez de disponibilidade e o baixo custo de obtenção são critérios recomendáveis. Para além dos critérios, a criação de um indicador sugere a atribuição de uma meta, a qual consiste na definição de um valor pretendido ao indicador em determinadas condições.

Já a análise de indicadores, conforme destaca Takashina (1999), consiste em extrair dos dados e resultados o seu mais amplo significado, para apoiar a avaliação do progresso e as tomadas de decisões. Além disso, o acompanhamento dos indicadores pode acorrer por comparações internas ou externas, observando-se a correlação das causas e efeitos entre os indicadores.

Para Mourão (2006), pode-se identificar um indicador como uma estatística, um fato, uma medida, uma série quantitativa de dados (indicador quantitativo) ou uma série de evidências ou percepções postuladas sobre a realidade (indicador qualitativo). 
Scheerens (2004), Takashina (1999) e Mourão (2006) conjugam da noção de que os indicadores são representações quantificáveis de determinado processo. Portanto permitem formular juízo de valores sobre o entendimento de qualidade e desempenho na educação, sendo utilizados para auxiliar nos processos administrativos ou na comparação entre momentos distintos de um mesmo processo ou, entre instituições, em comparação a padrão tido como ideal. Os autores também concordam que a estatística e os indicadores são instrumentos para se aproximar da realidade. Mas, assim como a teoria, a estatística não consegue englobar todos os aspectos envolvidos, por exemplo, em um processo educacional, quanto mais na sua universalidade social, política e econômica. Os indicadores são uma descrição, mas não a realidade. Os indicadores fazem partições da realidade para estudá-la. Ao voltar à realidade, é preciso reincorporar os aspectos extraídos, situando-os no espaço e tempo.

Medir não é fácil, embora inúmeros aspectos sejam tangíveis. A dificuldade aumenta quando se quer medir coisas intangíveis, tais como conhecimento, cultura científica, qualidade, inovação e impactos. Embora essa seja a nova tendência dentro dos indicadores, ainda não há padrões para tais medidas.

Norteando a questão dos indicadores está a definição do que se quer medir: a estatística sempre depende de definições, sendo que um esforço maior é requerido quanto mais subjetivo e intangível for o que se pretende medir. Definições são as bases para qualquer medida.

\section{OS MUNICÍPIOS CATARINENSES: IDEB, COEFICIENTE DE GINI E ÍNDICE DE POBREZA}

Localizado na Região Sul do país, o Estado de Santa Catarina possui uma população de mais de 6 milhões de habitantes, segundo o Censo 2010, distribuídos em 296 municípios, dos quais 231 têm menos de 20 mil habitantes e, entre estes, 109 não ultrapassam 5 mil, o que corresponde a mais de 33\% dos municípios catarinenses.

No entanto, esses dados não revelam as especificidades e contextos de produção dos indicadores sociais e educacionais 
das diferentes realidades que envolvem cada município, região ou grupos de municípios. Dessa forma, mesmo que gestores municipais, diretores de escolas e professores tenham acesso a inúmeros dados sobre suas cidades e comunidades, poucos significados são atribuídos a estes.

O presente estudo busca problematizar, a partir da reflexão anterior, o Índice de Desenvolvimento da Educação Básica (Ideb), indicador parcial da qualidade da educação, no contexto da realidade dos pequenos municípios catarinenses com população inferior a 5 mil habitantes, por meio do cruzamento com outros indicadores sociais (Índice de Pobreza e Coeficiente de Gini). O recorte temporal foi delimitado a partir dos ciclos da Prova Brasil - 2005, 2007 e 2009 - e selecionados apenas os municípios que participaram dos três ciclos e que possuíam, em 2010, menos de 5 mil habitantes.

Considerando-se que os três ciclos da Prova Brasil fornecem uma série histórica do Ideb, foram analisadas as variações do índice dividindo os municípios em dois grupos: 1 - elevação constante, indiferente ao grau, partindo de 2005 para 2007 e 2009; 2 - aumento do primeiro para o segundo ciclo com redução no terceiro, ou diminuição do primeiro para o segundo e aumento no terceiro. A figura 1 mostra a representação gráfica dos dois grupos.

FIGURA 1 - Representação gráfica dos dois grupos de municípios em relação ao desempenho do Ideb nos ciclos da Prova Brasil de 2005, 2007 e 2009

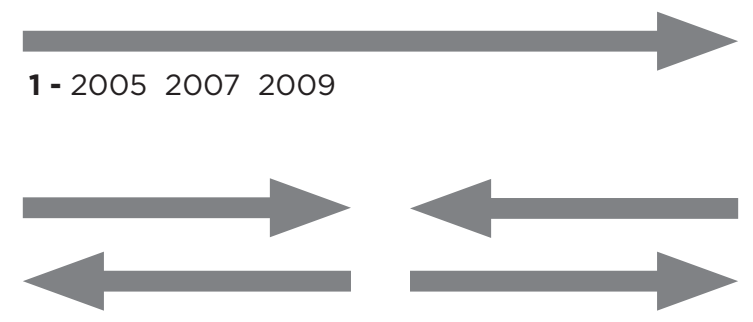

2 - 200520072009

Dos 109 municípios catarinenses com população inferior a 5 mil habitantes, 40 participaram dos três ciclos da Prova Brasil, constituindo a população do presente estudo. Destes, 33 apresentaram evolução do Ideb nos três ciclos, formando o Grupo 1. Apenas um teve o Ideb aumentado do ciclo de 2005 para 2007 e se manteve no mesmo índice em 2009. Os outros 
seis municípios ficaram divididos igualmente entre os que obtiveram melhor resultado em 2007 e os que tiveram pior resultado em 2007, compondo o Grupo 2.

GRÁFICO 1 - Distribuição dos municípios catarinenses com população inferior a 5 mil habitantes nos grupos 1 e 2

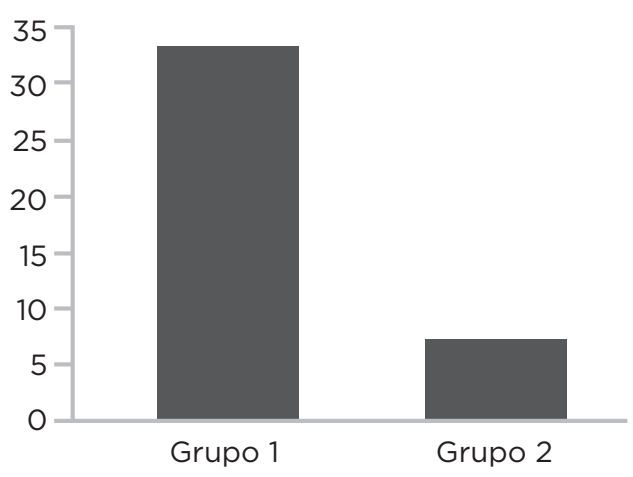

Fonte: IBGE. Censo Demográfico 2010. Ministério da Educação. Elaborado a partir dos dados da pesquisa.

Observando o gráfico 1, verifica-se a predominância dos municípios que tiveram aumento no Ideb em toda a série histórica.

Para fomentar o debate sobre os indicadores na realidade dos pequenos municípios catarinenses, foram levantados os Coeficientes de Gini para os 40 municípios e realizada a média de cada grupo. O Coeficiente de Gini é uma medida de desigualdade desenvolvida pelo matemático e estatístico italiano Corrado Gini e publicada, em 1912, no documento Variabilidade e mutabilidade. Ele consiste em um número entre 0 e 1, em que 0 corresponde à completa igualdade de renda e 1, à completa desigualdade. O índice de Gini é o coeficiente expresso em pontos percentuais.

Considerando essa média, tem-se o seguinte retrato: o Grupo 1, formado por 33 municípios que tiveram aumento do Ideb em toda a série histórica, apresenta uma média de 33,0 no Coeficiente de Gini; os demais municípios pertencentes ao Grupo 2 ficaram com 33,3 de média. Ou seja, verifica-se uma média do Coeficiente de Gini levemente superior nos municípios que tiveram variação negativa do Ideb no ciclo de 2007 ou de 2009. 
GRÁFICO 2 - Coeficiente de Gini dos municípios catarinenses com população inferior a 5 mil habitantes nos grupos 1 e 2

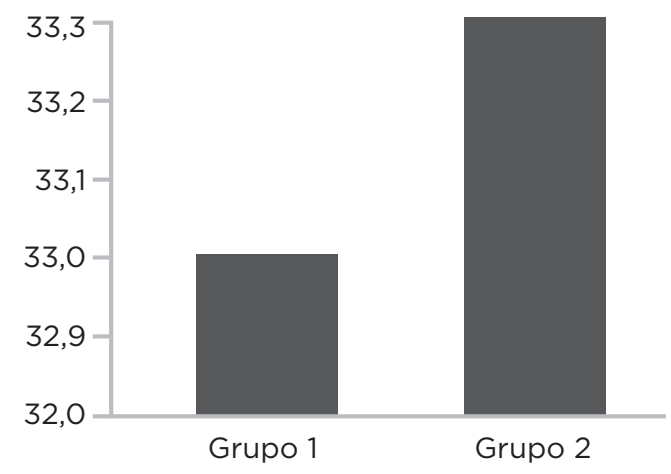

Fonte: IBGE. Censo Demográfico 2010. Ministério da Educação. Elaborado a partir dos dados da pesquisa.

Isso significa que, na média, os municípios do Grupo 1 têm menor desigualdade na distribuição de renda do que os do Grupo 2. Tal resultado poderia nos remeter à afirmação de que os municípios com menor desigualdade social teriam melhor rendimento escolar, já que são estes os que apresentaram variações positivas em todos os ciclos. Entretanto essa afirmação é frágil diante da complexidade que envolve os dois indicadores. Por um lado, o Ideb representa a parte mensurável de alguns aspectos considerados na qualificação da educação, mas não corresponde à realidade, traduzindo-se numa representação reduzida ou resumida desta, que não considera as diferenças sociais, políticas e econômicas dos alunos avaliados nos testes. Já o Coeficiente de Gini busca medir a diferença na distribuição de renda, que, neste caso específico, tem uma variação pequena, com amplitude de 0,06 pontos, ou seja, o menor e o maior Coeficiente de Gini são de, respectivamente, 0,30 e 0,36. Essa análise leva à questão do uso de médias para qualificar um processo ou fato social. Tanto o Ideb quanto o Coeficiente de Gini dos municípios são médias. Assim, qualquer afirmação baseada nesses índices representa apenas parte da realidade e não a sua totalidade. Ao compararmos esses dois indicadores estamos comparando partes sem que estes sejam reintegrados às suas realidades, caracterizando a fragilidade dos dados.

A partir dos mesmos grupos e metodologia, ou seja, os 33 municípios que tiveram aumento no Ideb em toda a série histórica e o grupo de seis municípios com redução do Ideb 
no ciclo de 2007 ou de 2009, passa-se, agora, a comparar os resultados com o Índice de Pobreza desenvolvido pelo IBGE. Neste caso os valores ficaram ainda mais próximos. O Grupo 1 apresentou média de $25,105 \%$ de pobres no conjunto dos municípios e o Grupo 2 registrou média de 25,218\%.

Mais uma vez o conjunto de municípios que não mantiveram crescimento constante do Ideb na série histórica apresentou piores condições sociais do que o grupo com regularidade de crescimento desse índice.

Essa análise, comparando o Ideb seja com o Coeficiente de Gini, seja com o Índice de Pobreza, é facilmente questionável em função da fragilidade estatística ou da falta de rigidez nas análises. Mas o objetivo deste estudo está exatamente em debater as fragilidades dos indicadores quando colocados à prova na sua reinserção na realidade da qual foram abstraídos.

O primeiro problema está no fato de que os indicadores são médias e, dessa forma, desconsideram as especificidades de cada unidade avaliada. A ideia de utilizar a média para comparação está diretamente ligada a essa fragilidade, pois este estudo pode ser facilmente criticado por empregar apenas as médias do Coeficiente de Gini e do Índice de Pobreza para populações muito diferentes em número: no caso, o Grupo 1 com 33 municípios e o Grupo 2 com apenas seis. No entanto, tal disparidade também não é considerada na construção dos indicadores, prevalecendo as médias, o que implica possíveis vieses nos resultados. Na contramão da crítica, este estudo considera a população de municípios catarinenses que participaram dos três ciclos da Prova Brasil e, assim, a diferença entre os grupos se justifica por abrangerem a totalidade dos casos estudados, compondo uma população de análise.

Ainda que não seja possível afirmar a relação categórica entre o Coeficiente de Gini, o Índice de Pobreza dos municípios e a regularidade no aumento do Ideb nos pequenos municípios catarinenses, esses dados indicam uma relação entre condições socioeconômicas e o rendimento escolar, contrariando o estudo Equidade entre as escolas das redes municipais - Uma análise com base nos dados de IDEB para municípios e escolas, desenvolvido pelo movimento Todos pela Educação, que foi realizado em 12 capitais e dez municípios paulistas com mais de 15 escolas que tiveram nota superior à média nacional no Ideb. Este estudo 
afirma que não há uma relação clara entre iniquidade na educação e desigualdade socioeconômica.

Há que se considerar que o estudo do movimento Todos pela Educação analisa um grupo de municípios diferente dos que são analisados neste artigo. Isso reforçaria a necessidade de ampliação das abordagens sobre indicadores nos pequenos municípios, pois aqui é possível perceber a existência de relação entre desigualdade social mensurada pelo Coeficiente de Gini, o Índice de Pobreza dos municípios e o Ideb, ainda que essa linha seja muito tênue. O fato revela que os resultados de estudos direcionados para os grandes municípios não podem ser generalizados para todo o Brasil.

O resultado principal desta análise é a indicação da fragilidade dos indicadores sociais e educacionais quando são efetuados cruzamentos simples. Sendo provenientes de uma mesma realidade, os indicadores necessitam de estudos aprofundados para significação dos dados mensuráveis diante da realidade de cada município ou região. No entanto isso não significa que esses estudos possam ser ignorados, pois apontam questões importantes e, quando contextualizados, podem contribuir para a qualificação de processos administrativos e pedagógicos.

\section{REFERÊNCIAS BIBLIOGRÁFICAS}

BAUER, R. Social indicators. Cambridge: MIT Press, 1967.

CARLEY, M. Indicadores sociais: teoria e prática. Rio de Janeiro: Zahar, 1985.

COLMAN, D.; NIXSON, F. Desenvolvimento econômico: uma perspectiva moderna. Rio de Janeiro: Campus, 1981.

HAKKERT, R. Fontes de dados demográficos. Belo Horizonte: ABEP, 1996.

JANNUZZI, P. de M. Indicadores sociais no Brasil: conceitos, medidas e aplicações. 3. ed. Campinas: Alínea; Campinas: PUC, 2004.

MOURÃO, P. Contributo para o estudo económico dos indicadores regionais. Departamento de Economia/Núcleo de Investigação em Políticas Económicas, Universidade do Minho. Disponível em: <http://www3.eeg.uminho.pt/ economia/nipe/docs/Publicações_Outrass_Revistas/com\%20Arbitragem/2006/ Mourão_2006_RPER.pdf>.

SCHEERENS, J. Melhorar a eficácia das escolas. Lisboa: ASA, 2004.

SEN, A. Desenvolvimento como liberdade. São Paulo: Companhia das Letras, 2005. 
TAKASHINA, N. T. Indicadores da qualidade e do desempenho. Rio de Janeiro: Qualitymark, 1999.

TRZESNIAK, P. Indicadores quantitativos: reflexões que antecedem seu estabelecimento. Ciência da Informação, v. 27, n. 2, p. 159-164, 1998. Disponível em: <http://www.scielo.br/pdf/ci/v27n2/2729808.pdf>. Acesso em: 28 abr. 2011.

USA. Department of Health, Education and Welfare. Toward a social report. Washington, D. C.: Government Printing Office, 1966.

\section{VALDECIR SOLIGO}

Doutorando em Educação pela Universidade do Vale do Rio dos Sinos (Unisinos), São Leopoldo - RS; Professor colaborador na Universidade Estadual do Oeste do Paraná (Unioeste), Foz do Iguaçu - PR valdecir_soligo@yahoo.com.br 